\title{
A Low/High Band Highly Linearized Reconfigurable Down Conversion Mixer in 65nm CMOS Process
}

\author{
Nisha Gupta \\ IIT Hyderabad \\ Email: 31.nisha@gmail.com
}

\author{
Ashudeb Dutta \\ IIT Hyderabad \\ Email: asudeb_dutta@iith.ac.in
}

\author{
Shiv Govind Singh \\ IIT Hyderabad \\ Email: sgsingh@iith.ac.in
}

\begin{abstract}
This paper presents a universal down conversion mixer for a multistandard wireless receiver with adapted reconfigurability in the form of RF bandwidth, active/passive and IF bandwidth. In the proposed architecture RF bandwidth reconfigurability is reconfigured between low band (LB) RF frequency and high band (HB) RF frequency mixer modes. LB / HB reconfigurability is made through power switching the transconductance amplifier. Active / Passive reconfigurability is made through switching the input signal between gate and source terminal of input transistors and enabling/disabling the transimpedance stage at the output. The CMOS transmission gate (TG) switches are designed to provide optimum headroom in this low voltage design. The proposed circuit is designed in the UMC $65 \mathrm{~nm}$ RFCMOS technology with $1.2 \mathrm{~V}$ supply voltage. From the simulation results, the proposed circuit shows conversion gain of $22 / 26 \mathrm{~dB}$ and $25 / 31 \mathrm{~dB}$, noise figure of $14.2 / 12.1 \mathrm{~dB}$ and 11.5/8.16 $\mathrm{dB}$, IIP3 of 10/8.1 dBm and $6.4 / 3 \mathrm{dBm}$ in $\mathrm{LB}$ and $\mathrm{HB}$ respectively where all these figures suggest passive/active mode. Hence this circuit will be much helpful in multi-standard receiver design.
\end{abstract}

\section{INTRODUCTION}

With increasing demand, communication technology also needs improvement. In communication Low [1] and high RF frequency applications are having different power demands. So wideband receiver is also a wastage of power. On the other hand Internet of Things (IoT) enabled platform also demands multi-mode multi-standard transceiver enhance the performance through seamless connectivity between zigbee, bluetooth, Wi-Fi, UWB and cognitive radio interface. To meet above demand most of the smart systems already support an increasing number of radios, which turns into a significant increase in component count and cost[2]-[5]. Therefore, the easiest solution is a single re-configurable radio. To make the radio re-configurable researchers introduce the RF frontend transceiver with reconfigurable LNA[6]-[7], PA, PLL[8], mixer[9]-[10], and filter[11] etc. Most of the proposed reconfigurable mixer have shown gain variability and Bandwidth tuning[12]-[13] through current variation, load tuning etc[9]-[11]. However, in multi-mode IoT systems, the other performances like noise figure, linearity reconfiguration need to be incorporated in mixer design. So our emphasis is to design a Mixer that can provide reconfigurability on these performances along with RF and IF bandwidth selection. In this regard, we propose a re-configurable down conversion mixer which switches between LB / HB and Active/Passive modes depends on performance requirement. Optimization between power can be achieved through switching between high and low frequencies.

The prime features of the re-configurable down conversion

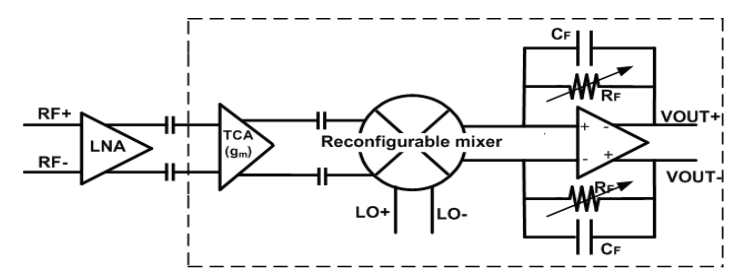

Fig. 1. Wide-band receiver front end.

mixer are:

1) Reconfiguration between low band and high band with gain and noise tunability at IF stage.

2) Power switching is used to switch on or off Transconductance amplifier.

3) Reconfiguration in the single circuitry between active and passive modes is done to chose the mixer according to desired performance requirement.

Upto the best of our knowledge, first time one such work is being reported in IoT perspective and detail operation are described in following paragraphs as section II details the proposed architecture and simulation results are explained in Section III.

\section{ARCHITECTURE}

The block diagram of wide-band RF front-end (demodulator) is shown in Fig. 1. The mixer is located after the LNA and downconverts the RF signal to a low IF signal. So it is required to design high input impedance $g_{m}$ stage to avoid the loading effect and to provide high isolation between IF, LO and RF. Widely used active mixer suffer from poor linearily, especially when the supply voltage is low. The opposite side passive mixer can provide very good linearity with comparable noise performance. So according to demand Active/Passive reconfigurability is done. The differential ended RF input is taken by RF balun with $50 \mathrm{ohm}$ input impedance termination. All the signal paths are fully differential in style to suppress the common mode noise and second order harmonic.

Reconfigurable front end mixer consists of two transconductance amplifier (LB / HB), two types of mixer (Active / Passive) in single circuitry and load stage. Transconductance amplifier that converts the RF input voltage into current is DC decoupled to switching stage. The mixer core output is directly coupled to transimpedance stage. IF voltage is built at the output of transimpedance amplifier after the RF current gets commutated in switching stage and passes to first order 


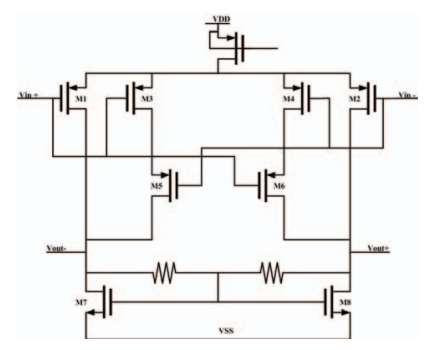

(a) Low Band TCA

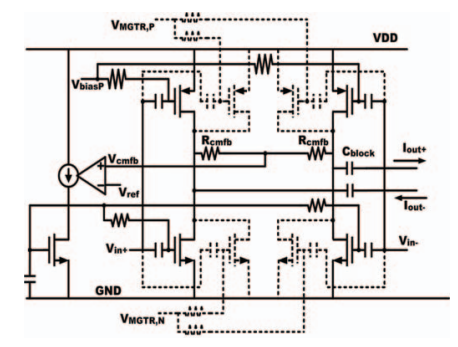

(b) High Band TCA
Fig. 2. Switch implementation using MOS

RC low pass filtering. TIA offers a virtual ground node for the $g_{m}$ stage, resulting in high linearity. Unlike narrow-band designs, $C_{P A R}$ (parasitic capacitance at the output node of the transconductance stage) is minimized to allow a less stringent noise specification upon the op-amp which favourably gets translated into lower power consumption circuit design.

\section{A. Transconductance Amplifier}

A current switching passive mixer consists of transconductance stage, switching stage and transimpedance amplifier stage. Fully differential CMOS transconductors are employed for $\mathrm{LB}$ and $\mathrm{HB}$ to convert the input RF voltage signal to RF current as shown in Fig. 2. Post which the current signal is fed to the switching stage ensuring that second order nonlinearity is reduced by using fully differential topology. TCA is modeled to minimize the signal loss and enhance its linearity performance.

Fig. 2(a) shows a simplified circuit schematic of the $g_{m}$ stage, where the main transistor pair M1 and M2 and auxiliary circuit M3-M6 are designed using PMOS that cancel the third order distortion of the main pair [1]. The $g_{m}$ stage is designed to dissipate $2.7 \mathrm{~mA}$ current from a $1.2 \mathrm{~V}$ supply with .1 to $1.5 \mathrm{G}$ bandwidth.

Both PMOS and NMOS input stages use balanced input devices with no tail current as shown in Fig. 2(b). The use of multi-gated [14] input pair allows tuning to be done to achieve higher IIP3 with almost same bias current. HB $g_{m}$ stage is designed to work from .6 to $5 \mathrm{GHz}$. and dissipate $5.2 \mathrm{~mA}$ current.

The common mode voltage is designed at VDD/2 for getting maximum swing. By setting VDD/2 common mode voltage, current can be minimized and parasitic capacitance at the output nodes of transconductor is optimized to the smallest possible value, thereby increasing the output impedance, for the purpose of improving the noise figure. The transconductance stage gain also reduces the overall noise of front end receiver.

\section{B. Active / Passive Mixer}

In this embodiment, modulator circuit is reconfigured between active and passive modes by switching between the output load, DC power supply and Gm stage (Gm MOS of active case) and current source shown in Fig. 3. Common source configuration is chosen because of compatibility with cases.

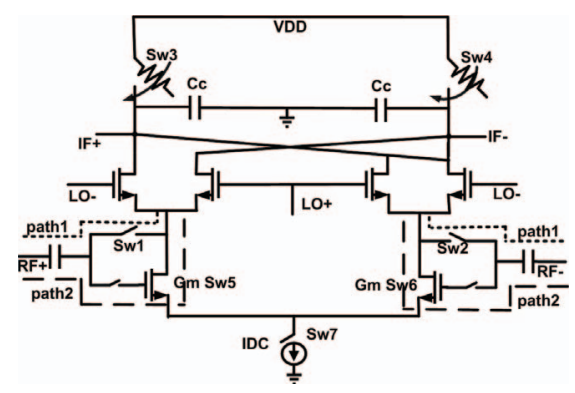

Fig. 3. Reconfigurable mixer.

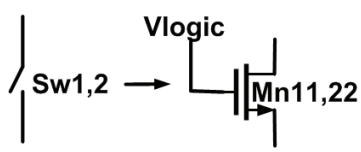

(a) NMOS switch

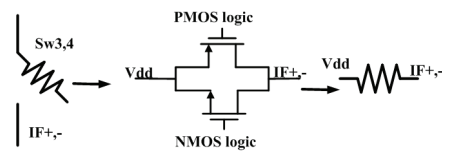

(b) resistive switch
Fig. 4. Switch implementation using MOS

In passive mode, the frequency mixer or modulator circuit is simply composed of four NMOS transistors characterized by resistance (Ron) when switched on. Accordingly, in order to make the common-source input stage configuration suitable for both active and passive mode topologies, switch (Sw12) are implemented using NMOS which have been added between the gate and drain of the common source transistor (Gm MOS). TCA differential output current is applied at the drain of the transistor Mn11 and Mn22 (NMOS switch 1-2) to route to the mixer core for mixing in the current domain. Vlogic high or low is given at the gate of Mn11 and Mn22 to configure reconfigurable mixer to operate in active/passive mode as shown in Fig. 4(a). Specifically when there is no current flowing through the mixer core, Vlogic is set to high, thus causing input signal to flow directly through the switching stage as shown in path 1 to mix with LO signal. Width of NMOS is chosen to provide degeneration resistance, thus turning the overall mixer topology into a passive mode as shown in Fig. 5(a). Transistors thus operate as switch 1-2 as well as degeneration resistance $R_{d e g}$ (switch 1-2 resistance), thereby increasing linearity of passive mixer [5]. Capacitor Cc is a high-frequency compensation capacitor used to suppress the noise at higher frequency. The modulated signal is supplied from the mixer core without any load and directly coupled with transimpedance amplifier. The voltage conversion gain of passive mixer is

$$
V C G=\frac{2}{\Pi} * g_{m} * Z_{F}
$$

where $Z_{F}$ is the feedback impedance of transimpedance amplifier, $C_{F}$ parallel with $R_{F}$ and $g_{m}$ is the transconductance of transconductance amplifier.

Resistive switches 3-4 designed using transmission gate, made of PMOS and NMOS switch, are fully turned off so that drain of the switching MOS is directly connected to input MOS of transimpedance amplifier without any coupling capacitor. Switches 5-7 designed using NMOS will also be off when this circuit operates in passive mode.

In active mode, input stage of the frequency mixer or modulator circuit have a configuration that is a common source 


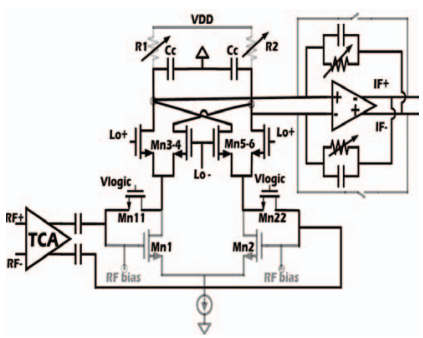

(a) Passive mode (LB or $\mathrm{HB})$

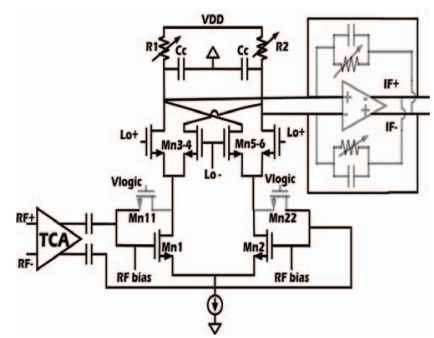

(b) Active mode (LB or HB)
Fig. 5. Reconfigurable mixer in passive and active mode

topology. This topology is chosen to provide better gain and low noise figure. Double balance gilbert cell architecture is used in active configuration when there is current flowing through input $(\mathrm{Gm})$ MOS Mn1 and Mn2 (Sw 5-6). The bias voltage can be selected to control parameters of input stage or switching operation of Gm MOS switch 5-6 as shown in path 2. The Gm of MOS Mn1 and Mn2 can be changed by changing the value of bias voltage, thus varying the gain of mixer. The optimum value of bias voltage is so desired so that mixer consumes a minimal amount of current. Switch 7 has been designed using NMOS which is biased in saturation region to provide current source. Thus turning the overall topology into an active mode as shown in Fig. 5(b).

Transmission gate is used as a resistive switch connected between VDD and IF output as shown in Fig. 4(b). W/L of PMOS and NMOS is chosen so that some voltage drop occurs across it and act as a resistance. Transmission gate total resistance is $R t o l=R_{P} M O S \| R_{N} M O S$. As it is connected to VDD so it acts as resistive load and Capacitor $\mathrm{Cc}$ is provided to act as a low pass filter in active mode operation of reconfigurable mixer. Gain of active mixer can be tuned by changing the resistance of transmission gate. The output of active mixer is directly passed to the output stage without going to TIA.

\section{Transimpedance Amplifier}

A simplified schematic of transimpedance amplifier is shown in Fig. 6(a). TIA consists of an operational transconductance amplifier with a feedback $R_{F} C_{F} . R_{F}$ and $C_{F}$ value is set according to IF frequency. A two stage miller compensated OTA topology is chosen for TIA design as shown in Fig. 6(b). First stage to provide high gain and second stage for high swing. So that structure can obtain both, high output swing and low input referred noise. Transimpedance amplifier is used to convert current to voltage output in passive mode operation. The TIA stage serves as load and anti-aliasing filter for the passive mixer. The TIA is designed in such a way so that very low impedance is provided at the passive mixer output. TIA input impedance is given by

$$
Z_{i n}(f)=\frac{2}{A(f)} * \frac{R_{F}}{1+2 \Pi R_{F} C_{F}}
$$

Where $A(f)$ is the open loop gain of the OTA. Due to high gain OTA bandwidth is limited and high frequency components suffer high impedance. In order to filter out high frequency components $C_{F}$ is inserted. This is done for all signal current to flow into feedback $R_{F} C_{F}$ from the mixer core. The TIA

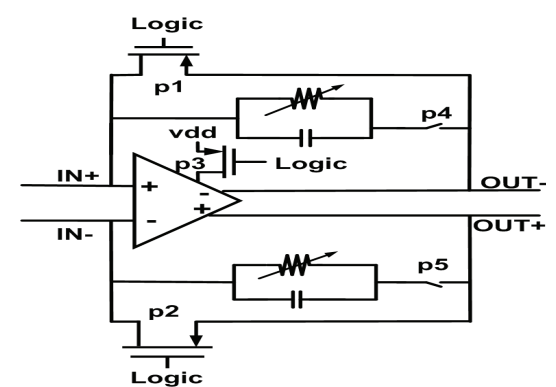

(a) Schematic of TIA stage

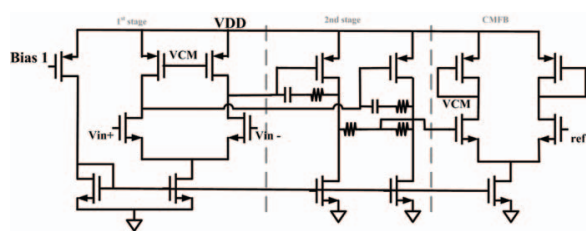

(b) Schematic of OTA

Fig. 6. Transimpedance amplifier

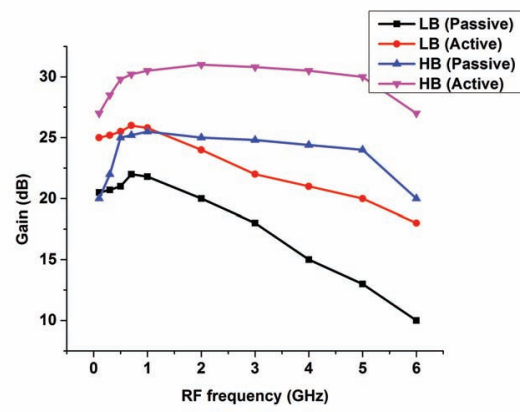

Fig. 7. Simulated conversion gain reconfigurable mixer vs RF frequency.

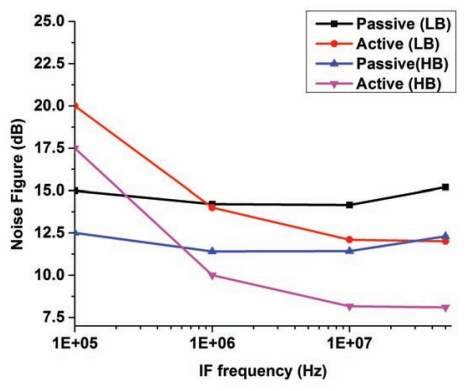

Fig. 8. Simulated noise figure vs IF frequency.

draws a total of $3.3 \mathrm{~mA}$ from the supply. In case of active mixer operation TIA will be switched off to save power. In case of active mixer operation TIA will be switched off by switching off p3 switch to save power and p1, p2 will be on. p4 and p5 also implemented using MOS and switch on or off in case of passive and active respectively. The gain of the TIA can be tuned by changing the value of $R_{F}$ and it provides another degree of freedom to configure the gain of the downconverter. 
TABLE I. SiMULATION RESULTS AND COMPARISON

\begin{tabular}{|c|c|c|c|c|c|c|c|c|c|c|}
\hline Parameters & Active(LB)(This work) & $\begin{array}{l}\text { Active (HB) } \\
\text { (This work) }\end{array}$ & $\begin{array}{l}\text { Passive (LB) } \\
\text { (This work) }\end{array}$ & $\begin{array}{l}\text { Passive (HB) } \\
\text { (This work) }\end{array}$ & [1] & [5] & [4] & [9] & [10] & {$[11]$} \\
\hline Gain $(\mathrm{dB})$ & 26 & 31 & 22 & 26 & $19.5-21$ & $22.5-25$ & 35 & $9-24$ & $1.2-17$ & $3.5-20.5$ \\
\hline Noise figure $(\mathrm{dB})$ & 12.1 & 8.16 & 14.2 & 11.4 & $11.4-12.4$ & $7.7-9.5$ & 10 & NA & $\geq 11$ & $\geq 8$ \\
\hline IIP3 (dBm) & 8.1 & 3 & 10 & 6.4 & $8-9$ & $\geq 7$ & 11 & 3.5 to -12 & 8.6 & $\leq 8.5$ \\
\hline Power(mW) & 7.17 & 10.66 & 7.05 & 10.56 & 5.4 & 10(mixer + TIA $)$ & 20.25 & 2.4 to 18 & 5.9 & $5.6-9.6$ \\
\hline Bandwidth(GHz) & .1 to 1.5 & .6 to 5 & .1 to 1.5 & .6 to 5 & $.048-.86$ & 1.55 to 2.3 & .7 to 2.5 & 2 to 10 & 1 to 12 & .7 to 2.3 \\
\hline Technology (CMOS) & $65 \mathrm{~nm}$ & $65 \mathrm{~nm}$ & $65 \mathrm{~nm}$ & $65 \mathrm{~nm}$ & $130 \mathrm{~nm}$ & $180 \mathrm{~nm}$ & $130 \mathrm{~nm}$ & $130 \mathrm{~nm}$ & $130 \mathrm{~nm}$ & $180 \mathrm{~nm}$ \\
\hline Power supply & $1.2 \mathrm{~V}$ & $1.2 \mathrm{~V}$ & 1.2 & 1.2 & 1.2 & 2 & 1.5 & 1.2 & 1.2 & 1.8 \\
\hline
\end{tabular}

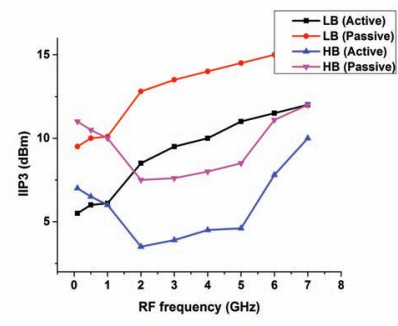

(a) $I I P_{3}$ of Reconfigurable mixer.

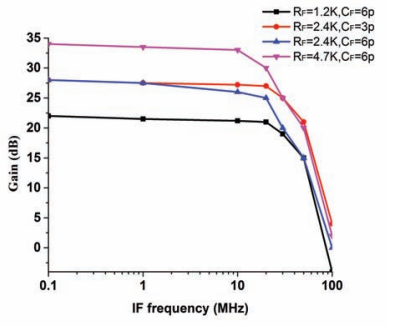

(b) voltage Conversion Gain of Reconfigurable mixer.

Fig. 9. Simulated linearity and Gain of reconfigurable mixer

\section{Simulation Results}

Based on the qualitative description of the building blocks, using their insights related to operation, the RF front-end demodulator is simulated in CMOS $65 \mathrm{~nm}$ process. The voltage conversion gain plot is shown in Fig. 7 with respect to RF frequency at $1 \mathrm{MHz}$ IF. The voltage conversion gain is close to $22 / 26 \mathrm{~dB}$ and $25 / 31$ for $\mathrm{LB}$ and $\mathrm{HB}$ case respectively where these figures suggest passive/active mode.

The simulated double side band noise figure at $800 \mathrm{MHz}$ and $2 \mathrm{GHz}$ for $\mathrm{LB}$ and $\mathrm{HB}$ respectively is shown in Fig. 8. The Simulated noise figure for active/passive is $14.2 / 12.1 \mathrm{~dB}$ and 11.5/8.16 dB @ 5MHz in LB and HB case respectively.

The two tone linearity test result is shown in Fig. 9(a) for $2 \mathrm{GHz}$ and $800 \mathrm{MHz}$ LO frequency for $\mathrm{HB}$ and $\mathrm{LB}$. Due to high conversion gain at low IF, the output compression point of the OPAMP, limits the input referred linearity of the circuit. $1 \mathrm{~dB}$-compression point of the circuit is limited by the output swing and varies with IF frequency. The simulated $I I P_{3}$ is $6.4 / 3 \mathrm{dBm}$ and $10 / 8.1$ for $\mathrm{HB}$ and $\mathrm{LB}$, where these figures suggest passive/active mode.

Fig. 9(b) depicts the voltage conversion gain of the proposed down conversion passive mixer of LB. By changing the value of $R_{F}$ and $C_{F}$ gain can be varied with constant bandwidth or by varying only $R_{F}$ conversion gain can be varied with little variation in bandwidth.

\section{CONCLUSiON}

In this paper, a wide band Reconfigurable mixer is simulated in $65 \mathrm{~nm}$ technology. A new concept of reconfiguration between Active / passive and LB / HB downconversion mixer is presented. This is an important design guideline for modern baseline deep sub-micron CMOS processes. Reconfigurable mixer is simulated in the frequency range $.1 \mathrm{GHz}$ to $6 \mathrm{GHz}$. The analytical results shows close to simulted results. With

the exception of few very stringent standard, the architechture is suitable for both cases.

\section{REFERENCES}

[1] Viet-Hoang Le; Hoai-Nam Nguyen; In-Young Lee; Seok-Kyun Han; Sang-Gug Lee, "A Passive Mixer for a Wideband TV Tuner," Circuits and Systems II: Express Briefs, IEEE Transactions on, vol.58, no.7, pp.398,401, July 2011

[2] Hong Zhang; Guican Chen; Xiao Yang, "Fully Differential CMOS LNA and Down-Conversion Mixer for 3-5 GHz MB-OFDM UWB Receivers," Radio-Frequency Integration Technology, 2007. RFIT 007. IEEE International Workshop on, vol., no., pp.54,57, 9-11 Dec. 2007.

[3] Chong-Ru Wu; Hsieh-Hung Hsieh et al., "A 3-5 GHz Frequency-Tunable Receiver Frontend for Multiband Applications," Microwave and Wireless Components Letters, IEEE, vol.18, no.9, pp.638,640, Sept. 2008.

[4] Poobuapheun, N.; Wei-Hung Chen; Boos, Z.; Niknejad, A.M., "A 1.5V $0.7-2.5 \mathrm{GHz}$ CMOS Quadrature Demodulator for Multi-Band DirectConversion Receivers," Custom Integrated Circuits Conference, 2006. CICC '06. IEEE, vol., no., pp.797,800, 10-13 Sept. 2006.

[5] Namsoo Kim; Aparin, V.; Larson, L.E., "A Resistively Degenerated Wideband Passive Mixer With Low Noise Figure and High IIP2 ," Microwave Theory and Techniques, IEEE Transactions on , vol.58, no.4, pp.820,830, April 2010.

[6] Xiaohua Yu; Neihart, N.M., "Analysis and Design of a Reconfigurable Multimode Low-Noise Amplifier Utilizing a Multitap Transformer," Microwave Theory and Techniques, IEEE Transactions on, vol.61, no.3, pp.1236,1246, March 2013.

[7] Geis, A.; Rolain, Y.; Vandersteen, G.; Craninckx, J., "A 0.045mm2 $0.16 \mathrm{GHz}$ reconfigurable multi-band, multi-gain LNA for SDR," Radio Frequency Integrated Circuits Symposium (RFIC), 2010 IEEE, vol., no., pp.123,126, 23-25 May 2010.

[8] Cook, B.W.; Berny, A.D.; Molnar, A.; Lanzisera, S.; Pister, K.S.J., "An Ultra-Low Power 2.4GHz RF Transceiver for Wireless Sensor Networks in $0.13 / \mathrm{spl} \mathrm{mu} / \mathrm{m}$ CMOS with $400 \mathrm{mV}$ Supply and an Integrated Passive RX Front-End," Solid-State Circuits Conference, 2006. ISSCC 2006. Digest of Technical Papers. IEEE International, vol., no., pp.1460,1469, 6-9 Feb. 2006.

[9] Min Wang; Saavedra, C.E., "Reconfigurable broadband mixer with variable conversion gain," Microwave Symposium Digest (MTT), 2011 IEEE MTT-S International, vol., no., pp.1,4, 5-10 June 2011.

[10] Jiangtao Xu, Saavedra, C.E.,"Guican Chen, A 12 GHz-Bandwidth CMOS Mixer With Variable Conversion Gain Capability," Microwave and Wireless Components Letters, IEEE Volume: 21, Issue: 10 Publication Year: 2011, Page(s): 565 - 567.

[11] Kuan Bao, Xiangning Fan et al.,"A CMOS Reconfigurable Passive Mixer with Digitally-Controllable Gain," Radio-Frequency Integration Technology (RFIT), 2014 IEEE International Symposium, Page(s): 1-3.

[12] R. Circa, D. Pienkowski, G. Bck, and R. Wittmann, Reconfigurable UMTS/WLAN RF receiver, in Proc. 3rd Software Radios Workshop, Karlsruhe, Germany, Mar. 2004, pp. 6571.

[13] Kakerow, R.; Mueller, M.; Pienkowski, D.; Circa, R.; Boeck, G., "Reconfigurable receiver approach for $4 \mathrm{G}$ terminals and beyond," IEEENEWCAS Conference, 2005. The 3rd International , vol., no., pp.9,12, 19-22 June 2005.

[14] Poobuapheun, N.; Wei-Hung Chen; Boos, Z.; Niknejad, A.M., "An inductorless high dynamic range $0.3 \quad 2.6 \mathrm{GHz}$ receiver CMOS frontend," Radio Frequency Integrated Circuits Symposium, 2009. RFIC 2009. IEEE , vol., no., pp.387,390, 7-9 June 2009 